MS05-P12

\section{Structure-assisted design of Carborane inhibitors of human Carbonic Anhydrase IX}

Pavlina Rezacova ${ }^{1}$, Michael Kugler ${ }^{1}$, Jiri Brynda ${ }^{2}$, Bohumir Gruner ${ }^{3}$, Marián Hajduch ${ }^{4}$, Jan Nekvinda ${ }^{3}$, Klara Pospisilova ${ }^{1}$, Viswanath Das $^{4}$, Josef Holub ${ }^{3}$

1. Institute of Organic Chemistry and Biochemistry, Czech Academy od Sciences, Prague, Czech Republic

2. Institute of Molecular Genetics, Czech Academy od Sciences, Prague, Czech Republic

3. Institute of Inorganic Chemistry, Czech Academy of Sciences, Rez, Czech Republic

4. Institute of Molecular and Translational Medicine, Olomouc, Czech Republic

email: rezacova@img.cas.cz

Carbonic anhydrases (CAs) are zinc metalloenzymes playing an important role in many physiological processes. Several CAs are also involved in various pathological processes in humans and represent thus targets for drug development. Specifically, human carbonic Anhydrase IX (CA IX), isoform overexpressed in solid hypoxic tumours, is a target for cancer therapy and diagnostics. We have previously identified carboranes as a promising class of specific inhibitors of CA IX [1]. Here we report recent advances in the structure-assisted design of carborane and metallacarborane inhibitors targeting CA IX. We chose carboranes, three-dimensional scaffolds, which act as as space-filling fragments. We modified boron cages to synthetize carboranes and metallacarboranes substituted by sulfamide, sulfonamide or sulfamate groups, i.e. functions known to bind tightly to the zinc atom in the active site of CAs. Consequently, the small library of ca. 70 substituted carboranes and metallacarboranes was. Several compounds exhibit selective inhibitory activity toward CAIX with $\mathrm{Ki}$ values in low nanomolar or even picomolar range. Selected inhibitors were tested for their effect on tumor growth in BALB/c mice orthotopically implanted with $4 \mathrm{~T} 1$ cells and SCID mice subcutaneous transplanted with HT-29 cells.

This work was supported by Czech Science Foundation, Project No. 15-05677S, Technology Agency of the Czech Republic, Projects No. TE01020028, and the Ministry of Education of the Czech Republic (LO1304 and EATRIS-CZ LM2015064 (programme "NPU I").

References:

1. J. Brynda, P. Mader, V. Šícha, M. Fábry, K. Poncová, M Bakardiev, B. Grüner, P. Cígler, P. Řezáčová, Angew Chem., Intl. Ed. Eng., 52, (2013), 13760.

Keywords: Anti-tumor agents, carborane inhibitors, Carbonic anhydrase IX

\section{MS05-P13}

\section{VHHs as tools in antibody assisted structure based drug discovery}

Sura Abbood ${ }^{1}$

1. Molecular and Cell Biology/ Leicester university, Leicester,
United Kingdom) email: sma43@leicester.ac.uk

Cytokines such as interleukin-6 (IL6) are attractive therapeutic targets in inflammatory diseases. Interleukin- 6 is a secreted cytokine, produced by different cell types, but it originates mainly from macrophages and monocytes at the site of inflammation [1]. IL6 is a multifunctional cytokine and mediates its biological function via a hexameric signaling complex composed of two molecules each of IL6, gp80 and gp130 [2]. It is a key player in immunological response and has been implicated in the pathogenesis of a wide range of inflammatory diseases such as rheumatoid arthritis [3]. Heavy chain only antibodies are produced by the immune system of a limited number of animals, including camels and llamas and due to their small size are an attractive tool for antibody based drug discovery. A number of antigen-binding domain (VHH) antibodies that target IL6 have developed to inhibit the hexameric signaling complex formation. VHH94 was derived from a camel heavy chain antibody isolated after immunization with a interleukin-6-gp80 fusion protein. VHH94 was found to enhance the binding of IL- 6 to gp80 potentially through binding to an allosteric site on IL-6 and partially inhibited the signaling of IL6. The combination of X-ray crystallography and NMR spectroscopy are using to provide a clear overall picture of the IL6-VHH94 interaction site.

References:

1. GABAY, C. (2006). Arthritis Research and Therapy, 8, Suppl 2:S3.

2. Boulanger, M. J. et al (2003). Science, 300, 2101-2104.

3. McInnes, I. B. \& Schett, G. (2007). Nat. Rev. Immunol., 7, 429 -442 .

Keywords: IL-6, VHH94, Structure 